\title{
Effect of Flaxseed Consumption on Central Obesity, Serum Lipids, and Adiponectin Levels in Overweight or Obese Women: A Randomized Controlled Clinical Trial
}

\author{
hoda ahmadniay motlagh ${ }^{1}$, erfaneh aalipanah ${ }^{1}$, mohsen mazidi ${ }^{2}$, and Shiva Faghih ${ }^{1}$ \\ ${ }^{1}$ Shiraz University of Medical Sciences \\ ${ }^{2}$ Chalmers University of Technology Department of Chemical and Biological Engineering
}

May 8, 2021

\begin{abstract}
Background: Flaxseed may be beneficial for the management of obesity due to its high content of alpha-linolenic acid, fiber, and lignans. Objective: We aimed to evaluate the effects of Flaxseed consumption on serum lipids, adiponectin, leptin, and weight loss in overweight or obese women. Methods: This randomized controlled clinical trial involved 60 overweight or obese women. Participants were randomly allocated to two groups; a) a balanced diet plus $30 \mathrm{~g} /$ day milled Flaxseed and b) a balanced diet plus 30g/day milled rice (as control group), for 12 weeks. Anthropometric indices, serum lipids, leptin, and adiponectin levels were measured at baseline and at the end of intervention. Results: After 12 weeks of intervention, there were significantly higher reductions in waist circumference $(\mathrm{WC})$ and waist to hip ratio $(\mathrm{WHR})$ (both $\mathrm{P}<0.05)$ in the flaxseed group compared to the controls. Moreover, adiponectin level was significantly increased in the flaxseed group (17.15 \pm 6.1$)$ compared to the controls $(16.83 \pm 10.5),(\mathrm{P}=0.001)$. However, there were no significant differences in serum lipid levels between the study groups before and after the intervention (all p $>0.05$ ). Conclusion: Flaxseed consumption may improve adiposity markers, as well as adiponectin levels Thus, flaxseed consumption could be an adjunctive therapy to attenuate central obesity. Serum lipid profile has not changed significantly after flaxseed consumption. Keywords: obesity, flaxseed, adiponectin, Leptin, blood lipids
\end{abstract}

Effect of Flaxseed Consumption on Central Obesity, Serum Lipids, and Adiponectin Levels in Overweight or Obese Women: A Randomized Controlled Clinical Trial

Background : Flaxseed may be beneficial for the management of obesity due to its high content of alphalinolenic acid, fiber, and lignans.

Objective : We aimed to evaluate the effects of Flaxseed consumption on serum lipids, adiponectin, leptin, and weight loss in overweight or obese women.

Methods : This randomized controlled clinical trial involved 60 overweight or obese women. Participants were randomly allocated to two groups; a) a balanced diet plus $30 \mathrm{~g}$ /day milled Flaxseed and b) a balanced diet plus $30 \mathrm{~g} /$ day milled rice (as control group), for 12 weeks. Anthropometric indices, serum lipids, leptin, and adiponectin levels were measured at baseline and at the end of intervention.

Results : After 12 weeks of intervention, there were significantly higher reductions in waist circumference (WC) and waist to hip ratio (WHR) (both $P<0.05$ ) in the flaxseed group compared to the controls. Moreover, adiponectin level was significantly increased in the flaxseed group (17.15 \pm 6.1 ) compared to the controls $(16.83 \pm 10.5),(\mathrm{P}=0.001)$. However, there were no significant differences in serum lipid levels between the study groups before and after the intervention (all $\mathrm{p}>0.05$ ).

Conclusion: Flaxseed consumption may improve adiposity markers, as well as adiponectin levels Thus, 
flaxseed consumption could be an adjunctive therapy to attenuate central obesity. Serum lipid profile has not changed significantly after flaxseed consumption.

\section{What's already known about this topic?}

the findings from previous clinical trials are still controversial. Therefore, we aimed to supplement whole flaxseed to understand its effects on serum adipokines (adiponectin and leptin),Anthropometric indices and lipid profile among overweight or obese women.

\section{What does this article add?}

our findings showed that flaxseeds could potentially reduce visceral obesity and decrease the risk of obesity through increasing adiponectin concentration.

Keywords : obesity, flaxseed, adiponectin, Leptin, blood lipids

\section{Introduction}

Obesity is one of the most important inflammatory diseases and its prevalence is increasing across the world (1). Obesity and overweight are associated with increased risk of several chronic disorders, and certain types of cancers (2).

It has been found that expression and secretion of adiponectin within the adipose tissue decreases in obese individuals (7). Adiponectin is the most prevalent adipokine which has beneficial effects on metabolism, fatty acids catabolism, low density lipoprotein cholesterol (LDL-C) oxidation, insulin sensitivity and suppression of inflammation $(5,6)$. Moreover, leptin has a energy balance regulation role, various hormonal functions as well as highly correlated to body fat mass $(8)$.

Flaxseed or linseed (Linum usitatissimum L. seed) known as a functional food contains healthy components such as alpha linolenic acid (ALA), lignans, dietary fibers and a variety of antioxidants and phytoestrogens $(12,13)$.

Flaxseed lignans has numerous health benefits. For instance, it can regulate expression and secretion of adipokines such as adiponectin and leptin (14-17). It has been shown that ALA has anti-inflammatory, antithrombotic and anti-arrhythmic effects $(16,18,19)$. ALA can deposit in adipose tissue where it may affect adipose tissue function and adipokine secretion (7). Besides ALA, flaxseed is a rich source of phytoestrogens (lignan) with potential benefits including cardio-protective and endocrine regulation effects $(20,21)$. These components are proposed to modify blood lipids levels mainly through regulating the gene expression of enzymes involved in the fatty acids metabolism (22-24). Previous studies in experimental models found an attenuating effect of flaxseed supplementation on serum lipids abnormalities and adiponectin and leptin secretion (25-28). However, the findings from clinical trials are conflicting $(29,30)$.

Therefore, in order to find the beneficial effects of whole flaxseed supplementation as an adjunct therapy to balanced diet on serum adipokines, body weight, Waist Circumference (WC) and lipid levels, we conducted the present trial on overweight or obese women.

\section{Methods}

\section{Study participants}

The present study was a randomized, double-blind, placebo-controlled clinical trial in which 60 overweight or obese women aged 25 to 50 years, with a body mass index (BMI) $25-35 \mathrm{~kg} / \mathrm{m}^{2}$ and regular menstrual cycles participated. Participants were excluded if they met one or more of the following criteria: 1) any chronic disease such as cardiovascular, renal, liver and infectious diseases as well as cancer, diabetes mellitus, and thyroid disorders, 2) with history of allergy to flaxseed, 3) being pregnant or lactating, 4) taking any medications that could affect lipid metabolism (Steroids, anti-hyperglycemic agents, statins), 5) taking supplements including multivitamins and minerals and also, herbal preparations, and 6) history of smoking or alcohol and drug abuse. The minimum sample size estimated for each group was 25 at a power (1- $\beta$ ) 
of $80 \%$ and $\alpha=0.05$ for a two-arm parallel study with two-tailed testing to detect a difference of $4 \mathrm{ng} / \mathrm{mL}$ in serum adiponectin concentration with a standard deviation of $5 \mathrm{ng} / \mathrm{mL}$, obtained from a previous study (31). Assuming a 10\% drop out, a total number of 60 participants was considered for this study. The study protocol, risks, and benefits were clarified

to the participants, and they signed a written informed consent at the time of enrollment. This study was approved by the Ethics Committee of Shiraz University of Medical Sciences, Shiraz, Iran (registration No. IR.SUMS.REC.1395.22) and was registered at Iranian Registry of Clinical Trials website (IRCT2016050327733N1).

\section{Study design}

The participants were randomly assigned to consume a balanced diet containing $30 \mathrm{~g} /$ day brown milled flaxseed (flaxseed group: $\mathrm{n}=30$ ) or $30 \mathrm{~g} /$ day raw milled rice (control group: $\mathrm{n}=30$ ) for 12 weeks. Random allocation was performed using blocked randomization method. The study participants, investigators, and outcome assessors were blinded to the type of interventions into which the individuals were allocated. The shape, color, and texture of milled rice were similar to the flaxseed product. For this purpose, we added edible colors to milled rice at the laboratory of pharmacy in Shiraz University of Medical Sciences. Calorie requirement of each subject was estimated using the estimated energy requirement (EER) equation (32). All diets consisted of $55 \%$ carbohydrates, $18 \%$ proteins, and $27 \%$ fats. Also, we provided a portion-size descriptive booklet of common foods for each participant. All the brown flaxseed products were purchased from registered herb provider Maleki Commercial Co. (Iran) and stored in a cool, dark, and dry place. They were milled within a week before delivery to the patients. Participants were asked to mix the powder with their dessert or daily meals (e.g., yogurt, salads, and soup) preferably for lunch. Moreover, participants were advised to maintain their usual physical activity during the intervention.

To assess the participants' adherence to the intervention, we asked them to bring back any unused flaxseed/placebo at each follow-up visit, so the investigators can estimate their adherence to supplementation during the study period. Participants were excluded if they consumed less than $90 \%$ of flaxseed/placebo. Follow-up assessments were done every 4 weeks (on 4th, 8th, and 12th weeks) in which participants were provided with enough supplement for the next four weeks. All measurements including anthropometric indices, blood levels of lipid profiles, leptin, adiponectin as well as dietary intakes and physical activities were performed at the baseline and at the end of the study (week 12).

\section{Assessment of anthropometric indices and blood lipid profile}

Anthropometric indices (height, weight, waist and hip circumferences) were measured for each participant at the baseline (week 0) and at the end of the study (week 12). Height was measured to the nearest $0.1 \mathrm{~cm}$ using a stadiometer (Seca 214 portable stadiometer) without shoes. Weight was recorded to the nearest $0.1 \mathrm{~kg}$ in light clothes, using a digital scale (Seca 881, Germany). BMI was calculated as body weight $(\mathrm{kg}) / \mathrm{height}$ squared $\left(\mathrm{m}^{2}\right)$. WC was measured to the nearest $0.1 \mathrm{~cm}$ by a non-stretch measuring tape in standing position, between the lower rib and iliac crest (33).

To assess the serum lipids, $5 \mathrm{cc}$ of blood sample was taken form participants after 12 hours of fasting at 7:00 8:00 A.M. The whole blood was centrifuged, and the obtained serum was kept at $-70^{\circ} \mathrm{C}$ until the end of study for the further analysis. Serum concentrations of lipid profile including Triglyceride (TG), Total Cholesterol (TC), LDL-C, and High Density Lipoprotein Cholesterol (HDL-C) were measured by the colorimetric method using commercial kits (Pars Azmoon Co., Iran). Serum leptin and adiponectin concentration were measured using ELISA (Enzyme-linked Immunosorbent Assay) by IBL kit (Parsazmun Co., Iran).

\section{Assessment of dietary intake and physical activity}

Energy and nutrients intake were estimated using 24-h dietary recalls for three nonconsecutive days $(2$ weekdays and one weekend day) and then were analyzed using Nutritionist IV (N-Squared Computing, San Bruno, CA, USA). Physical activity was evaluated using international physical activity questionnaire (IPAQ) 
for three days (two regular days and one weekend day). It was expressed as MET.h/day by multiplying the time of each physical activity by its relative Metabolic Equivalent Task (MET).

\section{Statistical methods}

Normality of each variable was tested by Kolmogorov-Smirnov. Pre- and post-treatment values were compared within groups using paired ttest. Between-group comparisons were made using independent samplet test. Effects of potential confounding factors were adjusted by the analysis of covariance. All ANCOVA models were adjusted for the baseline values of each variable and mean changes of BMI, WHR, physical activity, and energy intake. $\mathrm{P}<0.05$ was considered as statistically significant. SPSS version 22 (SPSS, Inc., Chicago, IL, USA) was used for analysis.

\section{Results}

A total of 100 women enrolled and after checking for eligibility, 60 individuals entered the study. During the follow-up, eight participants were excluded (one in the flaxseed group and seven in the control group) due to personal reasons. Finally, 52 subjects (Flaxseed group $n=29$ and control group $n=23$ ) completed the trial (Fig.1-Flowchart of participants through the study).

Baseline characteristics of participants are shown in (Table1). There were no significant differences in height, weight, BMI, waist and hip circumference of participants between flaxseed and control group at the beginning of the study.

No significant differences were observed between the two groups in mean daily intake of energy, protein, carbohydrate, fat, saturated fatty acids (SFAs), polyunsaturated fatty acids (PUFAs), mono unsaturated fatty acids (MUFAs), omega-6 fatty acids, at the beginning and the end of study. However, the amount of ALA and dietary fiber intake increased significantly in flaxseed group $(\mathrm{P}<0.001$ and $\mathrm{P}=0.01$ respectively). At the end of the study, ALA intake was significantly different between the two groups $(\mathrm{P}<0.001)$ (Table 2$)$.

As shown in Table 3, there was significant reductions in serum TG, TC, and LDL-C in the flaxseed group after 12 weeks of the intervention $(\mathrm{P}<0.001$ for all), whereas no significant decrease was observed in the control group. In addition, no significant changes were observed in serum HDL-C within each group after 12 weeks. There was no significant difference in serum TC, TG, LDL-C, and HDL-C between the study groups at baseline and at the end of study.

Moreover, weight, BMI, waist and hip circumference $(\mathrm{P}<0.001$ for all $)$ and WHR $(\mathrm{P}=0.003)$ decreased significantly in flaxseed group. Just a significant decrease was observed in hip circumference of the controls $(\mathrm{P}=0.004)$. Reduction of WC $(\mathrm{P}=0.001)$ and WHR $(\mathrm{P}=0.003)$ were significantly more in flaxseed group compared to the controls. After the follow up period, adiponectin concentration increased significantly in flaxseed group $(\mathrm{P}<0.001)$ which was statistically significant more than the controls $(\mathrm{P}=0.002)$. In addition, leptin concentration decreased significantly only in the flaxseed group, but this reduction was not statistically significant compared to the controls $(\mathrm{P}=0.29)$.

\section{Discussion}

We examined the effects of flaxseed consumption on anthropometric indices, serum leptin, lipids and adiponectin in overweight or obese women. Our findings indicated that the flaxseed group had significant reduction in weight and anthropometric indices such as waist and hip circumference and WHR. However, flaxseed consumption did not result in statistically significant effect on lipid profile compared to placebo.

Our data suggesting that flaxseed may have additional benefits on central adiposity compared with controls is in line with previous studies (16). Park et al. (34), found that flaxseed lignan (primarily secoisolariciresinol diglucoside) $[\mathrm{SDG}]$ might provide beneficial effects on obesity via reducing weight and fat accumulation. Wu et al. (35) showed that flaxseed supplementation $(30 \mathrm{~g} / \mathrm{d})$ for 12 weeks reduced central obesity, weight and waist circumference when combined with healthy lifestyle counseling. Conversely, Pindea et al. (36), reported no significant change in weight, BMI, and waist circumference following supplementation with 30 
$\mathrm{g} / \mathrm{d}$ flaxseed for 8 weeks. We assume that the duration of the study (less than 12 weeks) might be the reason why no significant anthropometric effects were observed (37).

The exact mechanisms by which flaxseed can ameliorate abdominal obesity remain unclear. Some studies have suggested that a diet rich in PUFAs may result in reduction of abdominal obesity (35), because increasing PUFAs in the diet might act as an important modulator for body fat deposition, Also according to previous studies, the high content of SDG can reduce or prevent obesity through increased fat oxidation $(3,38)$.

Very few randomized clinical trials have examined the effect of flaxseed supplementation on blood adiponectin and leptin concentration in people who are healthy $(31,39,40)$. Some of experimental studies reported remarkable effects of flaxseed on leptin and adiponectin $(3,7)$ and also altered circulating level of these hormone following supplementation with flaxseed $(7,23)$.

In the present study, the serum concentration of adiponectin increased significantly in the flaxseed group compared to control group. Cassani et al. (41) also showed that weight loss diet with $60 \mathrm{~g} / \mathrm{d}$ flaxseed supplementation in men with cardiovascular risk factors could improve adiponectin levels. Contrary to present study findings, Hutchins et al. (42), found no significant change in adiponectin levels with flaxseed supplementation in obese men and women with pre-diabetes. This contradictory finding might be due to the association of adiponectin with insulin resistance. According to other studies, response of adiponectin to an intervention might be quite different in an insulin-resistant population than in insulin-sensitive individuals (43-45). Furthermore, Nelson et al. (31) found a decrease in adiponectin levels of healthy overweight adults that were treated by flaxseed oil for 8 weeks which is consistent with our findings. The present study demonstrated that this effect might be attributed to a reduced demand for adiponectin's anti-inflammatory actions in face of high omega-3 fatty acids. In fact, in the case of adiponectin, the dosage of flaxseed given in different studies is critical, because the insulin-sensitizing and anti-inflammatory effects of ALA (the main component of flaxseed products) might deactivate the effects of adiponectin and result in a decreased demand for adiponectin (46). On the other hand, some researchers did not find significant correlation between PUFAs and circulating levels of adiponectin (47). So, they suggested ALA as an activator of peroxysomal proliferator activated receptor gamma (PPAR $\gamma)$ in adipocytes.

The adiponectin inducing effect of flaxseed might be because of its rich content of ALA. It seems that ALA is involved in increasing adiponectin secretion through stimulating transcription factors PPAR $\gamma(39)$. PPAR $\gamma$ is one of the key transcription factors which regulates adipogenesis and it can also control expressions of adiponectin, leptin and glucose transporter type 4 (GLUT4) (48). Moreover, SDG in flaxseeds could act as a PPAR $\gamma$ agonist and regulate adiponectin through an increase in PPAR $\gamma$ DNA binding activity in adipocytes (3). So, Flaxseed could probably be an effective component which can alter the metabolic process in adipose tissues in favor of lower visceral fat accumulation.

It should be noted that different outcome from flaxseed intervention may be related to inter-individual differences involved in the metabolic processes, resulting in altered adiponectin levels in circulation (15).

Previously high concentration of leptin in obese individuals has been reported in several studies $(10,49)$. We found reduction in serum leptin of both groups. However, this reduction was only significant in the flaxseed group. McCullough et al. (7) have found that leptin expression correlated positively to ALA content of flaxseed, so its effects on obesity related diseases might be due to a change in leptin expression, but in a study by Taylor et al. (37) which investigated the effect of dietary milled flaxseed and flaxseed oil on patients with type2 diabetes, leptin concentration did not change. Zhou et al (50) have suggested that insulin resistance might be correlated to depressed desaturase enzyme activity, so these patients are not able to transform ALA to Eicosapentaenoic Acid (EPA) and Docosahexaenoic Acid (DHA). This reason could probably why Taylor study could not show beneficial effect of omega-3 PUFAs from flaxseed on leptin levels.

Our findings that flaxseed consumption did not have a significant effect on lipid profile is consistent with that reported by Kaul et al., which showed that among apparently healthy adults, intake of $2 \mathrm{~g} /$ day flaxseed oil for 12 weeks had no significant effect on the lipid parameters in blood (51). In contrast, several clinical trials conducted in individuals with elevated levels of blood lipids, reported the beneficial effect of flaxseed 
supplementation on decreasing serum lipids (22, 52-55). Torkan et al. showed that consumption of $30 \mathrm{~g} / \mathrm{d}$ of flaxseed powder in hyperlipidemic patients for 40 days caused a significant decrease in TG, TC, and LDL-C compared to the placebo (54). Moreover, another clinical trial conducted in postmenopausal women with hypercholesterolemia showed a significant reduction in TC and LDL-C compared to the placebo following the flaxseed supplementation at the dosage of $30 \mathrm{~g} /$ day for 12 weeks (53). In the present study, the mean serum concentrations of lipids in both study groups were within the normal ranges, which could be the reason for disagreement of our results with previous studies. Not all of the clinical trials that were conducted on patients with hyperlipidemia, resulted in significant effects of flaxseed on blood lipids outcomes. Paschos et al. for example, found that the intake of $15 \mathrm{ml}$ of flaxseed oil for 12 weeks had no significant effect on lipid parameters in patients with dyslipidemia (56). Also, in a study among 62 individuals with baseline values of LDL-C between 130 and $200 \mathrm{mg} / \mathrm{dl}$, intake of $40 \mathrm{~g}$ /day of flaxseed-containing baked products for 10 weeks did not significantly change LDL-C and even caused a significant decrease in HDL-C in men, but not in women (57). Besides the initial serum lipids, it should be noted that some methodological differences such as small sample size, short duration of follow-up, type of the flaxseed product, the dosage of supplementation, and the degree of adherence to the intervention may be the possible reasons for the discrepancy between the studies.

Several mechanisms have been suggested for the beneficial effect of flaxseed on blood lipids. Flaxseed has a high content of ALA, an omega-3 fatty acid found in plants, as well as its highest amount of lignin among the plant foods (58). It has been shown that these compounds could reduce TC and LDL-C by reducing the gene expression of Sterol Regulatory Element-Binding Protein 1-C (SREBP-1c), which is involved in synthesis of fatty acids, and increasing the mRNA expression of Peroxisome Proliferator-Activated Receptor- $\alpha$ (PPAR- $\alpha$ ), which stimulates $\beta$-oxidation of fatty acids $(22,24,59)$. In addition, flaxseed is a rich source of dietary fibers, both soluble and insoluble. Dietary fiber is proposed to reduce blood cholesterol mainly through increasing bile acid excretion, synthesis of short-chain fatty acids, and insulin sensitivity (60).

This study has some important strengths including the use of an appropriate placebo for flaxseed. In addition, we used whole grain flaxseed instead of flaxseed oil or lignan. Nonetheless, our findings should be considered in light of several limitations. In particular, our sample size was small. Also, the present study cannot distinguish if the effects are due directly to ALA or lignin in flaxseeds. Moreover, whilst some studies found significant effect of flaxseed on lipid profiles, ours found no such effect. The possible reasons for non-significant results following the whole flaxseed products may be due to the differences in the quality of the test product, the amount of bioactive components and their bioavailability in the presence of some compounds such as glycosides and phytic acid (57) in the flaxseed products.

\section{Conclusion}

Overall, daily consumption of $30 \mathrm{~g}$ milled flaxseed for 12 weeks among overweight or obese women had no significant effect on blood lipid parameters. However, flaxseeds which contains PUFAs and lignan could potentially reduce visceral obesity and is therefore a promising food to help decrease the risk of obesity through increasing adiponectin concentration.

\section{Acknowledgment}

We thank the study participants for their involvement in this study. This manuscript funded by Shiraz University of Medical Sciences, Shiraz, Iran (grant no. IR.SUMS.REC.1395.22).

\section{Ethics}

All participants signed the written informed consent before initiating the study. The study was approved by the Ethics Committee of Shiraz University of Medical Sciences, Shiraz, Iran (registration no. IR.SUMS.REC.1395.22) and was registered at Iranian Registry of Clinical Trials website (IRCT2016050327733N1).

\section{REFRENCES:}


1. Woo JG, Guerrero ML, Ruiz-Palacios GM, Peng Y-m, Herbers PM, Yao W, et al. Specific infant feeding practices do not consistently explain variation in anthropometry at age 1 year in urban United States, Mexico, and China cohorts. The Journal of nutrition. 2013;143(2):166-74.

2. Vriz O, Lu H, Visentin P, Nicolosi L, Mos L, Palatini P. Gender differences in the relationship between left ventricular size and ambulatory blood pressure in borderline hypertension. The HARVEST Study. European heart journal. 1997;18(4):664-70.

3. Fukumitsu S, Aida K, Ueno N, Ozawa S, Takahashi Y, Kobori M. Flaxseed lignan attenuates high-fat diet-induced fat accumulation and induces adiponectin expression in mice. British journal of nutrition. 2008;100(03):669-76.

4. Matsuzawa Y, Funahashi T, Nakamura T. Molecular Mechanism of Metabolic Syndrome X: Contribution of Adipocytokines. Adipocyte-derived Bioactive Substances. Annals of the New York Academy of Sciences. 1999;892(1):146-54.

5. Haluzik M, Parizkova J, Haluzik M. Adiponectin and its role in the obesity-induced insulin resistance and related complications. Physiological Research. 2004;53(2):123-30.

6. Iacobellis G, Pistilli D, Gucciardo M, Leonetti F, Miraldi F, Brancaccio G, et al. Adiponectin expression in human epicardial adipose tissue in vivo is lower in patients with coronary artery disease. Cytokine. $2005 ; 29(6): 251-5$.

7. McCullough RS, Edel AL, Bassett CM, LaVallee RK, Dibrov E, Blackwood DP, et al. The alpha linolenic acid content of flaxseed is associated with an induction of adipose leptin expression. Lipids. 2011;46(11):104352 .

8. Zhou Y, Rui L. Leptin signaling and leptin resistance. Frontiers of medicine. 2013;7(2):207-22.

9. Beaulieu A, Poncin G, Belaid-Choucair Z, Humblet C, Bogdanovic G, Lognay G, et al. Leptin reverts proapoptotic and antiproliferative effects of $\alpha$-linolenic acids in BCR-ABL positive leukemic cells: involvement of PI3K pathway. PloS one. 2011;6(10):e25651.

10. Considine RV, Sinha MK, Heiman ML, Kriauciunas A, Stephens TW, Nyce MR, et al. Serum immunoreactive-leptin concentrations in normal-weight and obese humans. New England Journal of Medicine. 1996;334(5):292-5.

11. Park JB, Velasquez MT. Potential effects of lignan-enriched flaxseed powder on bodyweight, visceral fat, lipid profile, and blood pressure in rats. Fitoterapia. 2012;83(5):941-6.

12. Goyal A, Sharma V, Upadhyay N, Gill S, Sihag M. Flax and flaxseed oil: an ancient medicine \& modern functional food. Journal of food science and technology. 2014;51(9):1633-53.

13. Yari Z, Rahimlou M, Poustchi H, Hekmatdoost A. Flaxseed Supplementation in Metabolic Syndrome Management: A Pilot Randomized, Open-labeled, Controlled Study. Phytotherapy Research. 2016;30(8):1339-44.

14. Daleprane JB, Batista A, Pacheco JT, Da Silva AF, Costa CA, de Castro Resende Â, et al. Dietary flaxseed supplementation improves endothelial function in the mesenteric arterial bed. Food research international. 2010;43(8):2052-6.

15. Yang WS, Lee WJ, Funahashi T, Tanaka S, Matsuzawa Y, Chao CL, Chen CL, Tai TY, Chuang LM. Weight reduction increases plasma levels of an adipose-derived anti-inflammatory protein, adiponectin. J Clin Endocrinol Metab. 2001 Aug;86(8):3815-9.

16. Barre D, Mizier-Barre K, Stelmach E, Hobson J, Griscti O, Rudiuk A, et al. Flaxseed lignan complex administration in older human Type 2 diabetics manages central obesity and prothrombosis - an invitation to further investigation into polypharmacy reduction. Journal of nutrition and metabolism. 2012;2012. 
17. Blüher S, Mantzoros CS. Leptin in humans: lessons from translational research. The American journal of clinical nutrition. 2009;89(3):991S-7S.

18. Machado AM, de Paula H, Cardoso LD, Costa NM. Effects of brown and golden flaxseed on the lipid profile, glycemia, inflammatory biomarkers, blood pressure and body composition in overweight adolescents. Nutrition. 2015;31(1):90-6.

19. Khalatbari Soltani S, Jamaluddin R, Tabibi H, Yusof M, Nisak B, Atabak S, et al. Effects of flaxseed consumption on systemic inflammation and serum lipid profile in hemodialysis patients with lipid abnormalities. Hemodialysis International. 2013;17(2):275-81.

20. Bloedon LT, Szapary PO. Flaxseed and cardiovascular risk. Nutrition reviews. 2004;62(1):18-27.

21. Pan A, Yu D, Demark-Wahnefried W, Franco OH, Lin X. Meta-analysis of the effects of flaxseed interventions on blood lipids. The American journal of clinical nutrition. 2009;90(2):288-97.

22. Fukumitsu S, Aida K, Shimizu H, Toyoda K. Flaxseed lignan lowers blood cholesterol and decreases liver disease risk factors in moderately hypercholesterolemic men. Nutrition research (New York, NY). 2010;30(7):441-6.

23. Wang, M., Zhang, XJ., Feng, K. et al. Dietary $\alpha$-linolenic acid-rich flaxseed oil prevents against alcoholic hepatic steatosis via ameliorating lipid homeostasis at adipose tissue-liver axis in mice. Sci Rep 6, 26826 (2016).

24. Jump DB, Botolin D, Wang Y, Xu J, Christian B, Demeure O. Fatty acid regulation of hepatic gene transcription. The Journal of nutrition. 2005;135(11):2503-6.

25. Felmlee MA, Woo G, Simko E, Krol ES, Muir AD, Alcorn J. Effects of the flaxseed lignans secoisolariciresinol diglucoside and its aglycone on serum and hepatic lipids in hyperlipidaemic rats. The British journal of nutrition. 2009;102(3):361-9.

26. Park JB, Velasquez MT. Potential effects of lignan-enriched flaxseed powder on bodyweight, visceral fat, lipid profile, and blood pressure in rats. Fitoterapia. 2012;83(5):941-6.

27. Pellizzon MA, Billheimer JT, Bloedon LT, Szapary PO, Rader DJ. Flaxseed reduces plasma cholesterol levels in hypercholesterolemic mouse models. Journal of the American College of Nutrition. 2007;26(1):66-75.

28. Prasad K, Mantha S, Muir A, Westcott N. Reduction of hypercholesterolemic atherosclerosis by CDCflaxseed with very low alpha-linolenic acid. Atherosclerosis. 1998;136(2):367-75.

29. Cornish SM, Chilibeck PD, Paus-Jennsen L, Biem HJ, Khozani T, Senanayake V, et al. A randomized controlled trial of the effects of flaxseed lignan complex on metabolic syndrome composite score and bone mineral in older adults. Applied Physiology, Nutrition, and Metabolism. 2009;34(2):89-98.

30. Lemay A, Dodin S, Kadri N, Jacques H, Forest J-C. Flaxseed dietary supplement versus hormone replacement therapy in hypercholesterolemic menopausal women. Obstetrics \& Gynecology. 2002;100(3):495504.

31. Nelson TL, Stevens JR, Hickey MS. Adiponectin levels are reduced, independent of polymorphisms in the adiponectin gene, after supplementation with $\alpha$-linolenic acid among healthy adults. Metabolism. 2007;56(9):1209-15.

32. Dent E, Kowal P, Hoogendijk EO. Frailty measurement in research and clinical practice: A review. European journal of internal medicine. 2016;31:3-10.

33. Mahan LK, Raymond JL. Krause's Food \& the Nutrition Care Process-E-Book: Elsevier Health Sciences; 2016.

34. Park JB, Velasquez MT. Potential effects of lignan-enriched flax seed powder on blood pressure, fat accumulation, and lipid profile in rats. The FASEB Journal. 2011;25(1_MeetingAbstracts):339.6. 
35. Wu H, Pan A, Yu Z, Qi Q, Lu L, Zhang G, et al. Lifestyle counseling and supplementation with flaxseed or walnuts influence the management of metabolic syndrome. The Journal of nutrition. 2010;140(11):1937-42.

36. Pineda AMR, Gómez BLG, Alzate CMA, Castaño CFM, Marín BEL. Consumo de linaza molida para la reducción de peso corporal en personas con exceso de peso. Perspectivas en Nutrición Humana. 2011;13(1):56.

37. Taylor CG, Noto AD, Stringer DM, Froese S, Malcolmson L. Dietary milled flaxseed and flaxseed oil improve N-3 fatty acid status and do not affect glycemic control in individuals with well-controlled type 2 diabetes. J Am Coll Nutr. 2010 Feb;29(1):72-80.

38. Prasad K. Reduction of serum cholesterol and hypercholesterolemic atherosclerosis in rabbits by secoisolariciresinol diglucoside isolated from flaxseed. Circulation. 1999;99(10):1355-62.

39. Kratz M, Swarbrick MM, Callahan HS, Matthys CC, Havel PJ, Weigle DS. Effect of dietary n-3 polyunsaturated fatty acids on plasma total and high-molecular-weight adiponectin concentrations in overweight to moderately obese men and women. The American journal of clinical nutrition. 2008;87(2):347-53.

40. Hallund J, Ravn-Haren G, Bügel S, Tholstrup T, Tetens I. A lignan complex isolated from flaxseed does not affect plasma lipid concentrations or antioxidant capacity in healthy postmenopausal women. The Journal of nutrition. 2006;136(1):112-6.

41. Cassani RSL, Fassini PG, Silvah JH, Lima CMM, Marchini JS. Impact of weight loss diet associated with flaxseed on inflammatory markers in men with cardiovascular risk factors: a clinical study. Nutrition journal. 2015;14(1):5.

42. Hutchins AM, Brown BD, Cunnane SC, Domitrovich SG, Adams ER, Bobowiec CE. Daily flaxseed consumption improves glycemic control in obese men and women with pre-diabetes: a randomized study. Nutrition Research. 2013;33(5):367-75.

43. Patel JV, Lee KW, Tomson J, Dubb K, Hughes EA, Lip GY. Effects of omega-3 polyunsaturated fatty acids on metabolically active hormones in patients post-myocardial infarction. International journal of cardiology. 2007;115(1):42-5.

44. Weyer C, Funahashi T, Tanaka S, Hotta K, Matsuzawa Y, Pratley RE, et al. Hypoadiponectinemia in obesity and type 2 diabetes: close association with insulin resistance and hyperinsulinemia. The Journal of Clinical Endocrinology \& Metabolism. 2001;86(5):1930-5.

45. Flachs P, Mohamed-Ali V, Horakova O, Rossmeisl M, Hosseinzadeh-Attar M, Hensler M, et al. Polyunsaturated fatty acids of marine origin induce adiponectin in mice fed a high-fat diet. Diabetologia. 2006;49(2):394-7.

46. Babcock TA, Novak T, Ong E, Jho DH, Helton WS, Espat NJ. Modulation of lipopolysaccharidestimulated macrophage tumor necrosis factor- $\alpha$ production by $\omega-3$ fatty acid is associated with differential cyclooxygenase-2 protein expression and is independent of interleukin-10. Journal of Surgical Research. $2002 ; 107(1): 135-9$.

47. Jose-Manuel Fernandez-Real, Joan Vendrell, Wifredo Ricart, Circulating Adiponectin and Plasma Fatty Acid Profile, Clinical Chemistry, Volume 51, Issue 3, 1 March 2005, Pages 603-609.

48. Rosen ED, Walkey CJ, Puigserver P, Spiegelman BM. Transcriptional regulation of adipogenesis. Genes \& development. 2000;14(11):1293-307.

49. Hariri M, Ghiasvand R, Shiranian A, Askari G, Iraj B, Salehi-Abargouei A. Does omega-3 fatty acids supplementation affect circulating leptin levels? A systematic review and meta-analysis on randomized controlled clinical trials. Clinical endocrinology. 2015;82(2):221-8.

50. Zhou YE, Kubow S, Dewailly E, Julien P, Egeland GM. Decreased activity of desaturase 5 in association with obesity and insulin resistance aggravates declining long-chain n-3 fatty acid status in Cree undergoing dietary transition. British journal of nutrition. 2009;102(6):888-94. 
51. Kaul N, Kreml R, Austria JA, Richard MN, Edel AL, Dibrov E, et al. A comparison of fish oil, flaxseed oil and hempseed oil supplementation on selected parameters of cardiovascular health in healthy volunteers. J Am Coll Nutr. 2008;27(1):51-8.

52. Mandasescu S, Mocanu V, Dascalita AM, Haliga R, Nestian I, Stitt PA, et al. Flaxseed supplementation in hyperlipidemic patients. Revista medico-chirurgicala a Societatii de Medici si Naturalisti din Iasi. 2005;109(3):502-6.

53. Patade A, Devareddy L, Lucas EA, Korlagunta K, Daggy BP, Arjmandi BH. Flaxseed reduces total and LDL cholesterol concentrations in Native American postmenopausal women. Journal of women's health (2002). 2008;17(3):355-66.

54. Torkan M, Hassan Entezari M, Siavash M. Effect of flaxseed on blood lipid level in hyperlipidemic patients. Reviews on recent clinical trials. 2015;10(1):61-7.

55. Zhang W, Wang X, Liu Y, Tian H, Flickinger B, Empie MW, et al. Dietary flaxseed lignan extract lowers plasma cholesterol and glucose concentrations in hypercholesterolaemic subjects. British Journal of Nutrition. 2008;99(6):1301-9.

56. Paschos GK, Zampelas A, Panagiotakos DB, Katsiougiannis S, Griffin BA, Votteas V, et al. Effects of flaxseed oil supplementation on plasma adiponectin levels in dyslipidemic men. European journal of nutrition. 2007;46(6):315-20.

57. Bloedon LT, Balikai S, Chittams J, Cunnane SC, Berlin JA, Rader DJ, et al. Flaxseed and cardiovascular risk factors: results from a double blind, randomized, controlled clinical trial. J Am Coll Nutr. 2008;27(1):6574.

58. Singh K, Mridula D, Rehal J, Barnwal P. Flaxseed: a potential source of food, feed and fiber. Critical reviews in food science and nutrition. 2011;51(3):210-22.

59. Shimano H, Yahagi N, Amemiya-Kudo M, Hasty AH, Osuga J, Tamura Y, et al. Sterol regulatory element-binding protein-1 as a key transcription factor for nutritional induction of lipogenic enzyme genes. The Journal of biological chemistry. 1999;274(50):35832-9.

60. Brown L, Rosner B, Willett WW, Sacks FM. Cholesterol-lowering effects of dietary fiber: a meta-analysis. Am J Clin Nutr. 1999;69(1):30-42.

\section{Hosted file}

diagram.pdf available at https://authorea.com/users/412540/articles/521214-effectof-flaxseed-consumption-on-central-obesity-serum-lipids-and-adiponectin-levels-inoverweight-or-obese-women-a-randomized-controlled-clinical-trial

\section{Hosted file}

tables.pdf available at https://authorea.com/users/412540/articles/521214-effect-offlaxseed-consumption-on-central-obesity-serum-lipids-and-adiponectin-levels-inoverweight-or-obese-women-a-randomized-controlled-clinical-trial 